\title{
Interactions between coincident and orthogonal cues to texture boundaries
}

\author{
W. A. PHILUIPS and B. J. CRAVEN \\ University of Stirling, Stirling, Scotland
}

\begin{abstract}
Arrays with horizontal or vertical texture boundaries formed by element orientation and length cues were displayed, and the texture boundary formed by one cue was specified as the target. The boundaries formed by the two cues were coincident on some trials and orthogonal on others. Observers' accuracy in reporting the orientation of the target boundary was improved by a coincident nontarget boundary and was worsened by an orthogonal one. Conditional mutual information measures are used to show how effects due to contextual modulation can be distinguished from effects due to additive combination of the cues. The results of five experiments are interpreted as evidence that the transmission of information about specific texture boundary cues is modulated by task context but not by a coincident or orthogonal boundary in another cue. We therefore distinguish between the effects of "context," as shown by the effects of any variable not called the target, and "modulatory contextual effects," as shown by the effects of one variable on the transmission of information specifically about another.
\end{abstract}

To process and learn about sensory input, it is necessary to organize it into much smaller subsets that distinguish figures from ground. Texture boundaries are common in natural images, and they play an important role in figural grouping. Psychophysical evidence suggests that feature gradients are computed simultaneously in many distinct feature maps. Minimally, this involves two stages of filtering for each texture feature. The first detects the features (e.g., Gabor-like elements at a range of orientations and spatial scales), and the second computes the rate of change of feature activity across two-dimensional (2-D) spatial position (e.g., Beck, 1982; Landy \& Bergen, 1991; Nothdurft, 1993; Pashler, 1988; Sagi \& Julesz, 1985). The gradients of contrast in each of the separate feature dimensions may then be combined into a single generalized texture boundary map that shows the spatial distribution of feature contrasts without distinguishing the individual features that produce those contrasts (e.g., Atkinson \& Braddick, 1989; Sagi \& Julesz, 1985). This generalized texture boundary map could then be used as the basis for guiding attention to salient locations on that map (e.g., Nothdurft, 1992; Sagi, 1995; Scialfa \& Joffe, 1995; Wolfe, 1994).

Texture boundaries are usually signaled by multiple partially correlated cues, and computational algorithms for finding texture boundaries in natural images perform bet-

Work on this paper was supported in part by a Visiting Fellowship to W.A.P. at the Neurosciences Institute, San Diego. The authors thank Evan Balaban, Ranald Macdonald, Jeremy Wolfe, and three anonymous referees for helpful and insightful comments on an earlier version of this paper. Correspondence should be addressed to W. A. Phillips, Centre for Cognitive and Computational Neuroscience, Department of Psychology, University of Stirling, Stirling, FK9 4LA, Scotland (e-mail: wapl@stir.ac.uk). ter if they use multiple cues (Poggio, Gamble, \& Little, 1988). The parallel feature architecture outlined above computes gradients in many different texture cues simultaneously, and psychophysical studies suggest that multiple cues are used when available because performance is improved when different cues agree and is worsened when they disagree.

Given that multiple partially correlated cues contribute to texture segregation, the aims of this paper are (1) to show how additive and modulatory interactions between cues can be distinguished, (2) to determine whether either or both forms of interaction occur between the texture cues studied, and (3) to determine whether the processing of information about individual cues is modulated by the task context in which they occur. These aims require us to make the concept of contextual modulation clear, and we give explicit criteria for determining whether it occurs by using measures of conditional mutual information, as described below. The stimulus context that we study is that provided by a texture boundary specified by another cue, where that boundary is either coincident with or orthogonal to the target cue. The task context studied is whether or not the cue is specified as the target by the instructions given to the subject.

The basic idea underlying the distinction between additive and modulatory interactions is that additive interactions compute the value of a new higher level variable from the values of the input variables, whereas, in modulatory interactions, the modulatory variables affect the transmission of information specifically about other variables from which they remain distinct. The outputs of additive interactions thus transmit information about all of the inputs from which they are computed, but without keeping their individual contributions distinct. Computing a generalized texture boundary map is a good example of an 
additive interaction (Landy, 1993), as is that of localizing contours by additive combinations of luminance, color, motion, and texture (Rivest \& Cavanagh, 1996). In contrast to this, the output of a modulatory interaction transmits information about the modulated variable in a way that keeps it distinct from the modulatory variable. This implies a fundamental asymmetry between the effects of modulated and modulating variables. An example of modulation in early vision may be provided by the lateral interactions between elementary feature detectors in nearby but nonoverlapping regions of the visual field, for which there is both psychophysical (e.g., Polat \& Sagi, 1993) and electrophysiological (e.g., Polat, Mizobe, Pettet, \& Norcia, 1998) evidence. Zipser, Lamme, and Schiller (1996) show that the firing rates of cells in V1 of awake macaque monkeys are enhanced after about $80 \mathrm{msec}$ if their activity is part of the figure, rather than of the ground. This is interpreted as showing that higher level stimulus context can modulate the firing rate of elementary feature detectors, but without changing the features that they detect. This conception of contextual modulation also applies to the effects of attention, because attention to particular features can increase their salience without changing their identity. Stimulus and task contexts may therefore modulate signal processing in similar ways.

There are both theoretical and empirical grounds for studying interactions between texture boundary cues. Our primary theoretical motivation is that contextual modulation is central to recent theories of cortical function (e.g., Gilbert 1992; Phillips \& Singer, 1997; Tononi, Sporns, \& Edelman, 1992). The theoretical framework outlined by Phillips and Singer (1997) is based on a distinction between primary interactions that determine the content of any message transmitted within the cognitive system and modulatory interactions that affect transmission of that message but without becoming part of its content (e.g., by changing its salience or the time at which it is sent). Formal procedures for distinguishing empirically between additive and modulatory interactions have recently been developed and studied analytically (Smyth, Phillips, \& Kay, 1996). These techniques have general utility, and, here, we show how they can be used to design and analyze psychophysical studies of the interactions between multiple cues.

Another theoretical motivation for studying interactions between multiple cues is that relaxation labeling has been shown to be a useful technique in computer vision (e.g., Barrow \& Tenenbaum, 1978). Green (1991) reviews much evidence in support of the view that it occurs in human vision, including texture segregation. Relaxation uses constraints between different streams of processing to discover as coherent a set of outputs, or labels, as possible. The stimulus input to each stream constrains the features that it might signal as being present in that input. When two or more alternatives have a significant probability, then the input is ambiguous, and interactions between streams emphasize those alternatives that increase the coherence of the outputs as a whole. Modulatory interactions may therefore implement some form of relaxation labeling. Green notes that much of the relevant psychophysical data can also be accounted for by simple summation, however, so the role of modulatory interactions in human texture segregation remains an important and unresolved issue.

An empirical reason for studying modulation in texture segregation is that many studies show better performance when different cues agree and worse performance when they disagree. Furthermore, a few of these studies suggest that the interactions may be nonlinear, so it is worth asking to what extent these effects involve modulation. Green (1991) reviews many such studies, so, here, we summarize only a few particularly relevant examples. More recent discussion can be found in the studies of $\mathrm{Ri}$ vest and Cavanagh (1996) and Landy (1993), both of which provide further evidence for additive interactions between texture boundary cues.

Various studies have shown improvement in performance when different texture boundary cues agree. Beck (1967) showed that perceptual grouping is enhanced when both the form and the luminance of texture elements vary between two regions and that these variables interact. Callaghan, Lasaga, and Garner (1986) studied reaction times (RTs) to locate a distinct quadrant in $6 \times 6$ arrays of line elements that varied in orientation, color, or both. RTs were shorter when both cues agreed. Callaghan (1989) studied RTs to decide whether a central texture boundary in $6 \times$ 6 arrays was horizontal or vertical. RTs were reduced by having form cues agree with the hue cues, but only when the hue differences were difficult to detect. Better performance with multiple coincident cues has also been shown in visual search tasks in which observers must detect, locate, or identify single texture elements within an array (Farell, 1984; Nothdurft, 1993; Wolfe, Cave, \& Franzel, 1989).

Previous studies also show interference when different cues disagree. Callaghan (1984) studied RTs to segregate a $6 \times 6$ array into two halves differing in color. Irrelevant variation of brightness interfered with segregation judgments based on hue. Interference between orientation and color was shown by Callaghan et al. (1986) and between hue and form by Callaghan (1989). Variation of an irrelevant cue has also been shown to produce interference in visual search (Nothdurft, 1993; Pashler, 1988; Theeuwes, 1991, 1992).

A few studies suggest that there are nonlinear interactions between cues to texture segregation. Caelli and Moraglia (1985) studied the perception of textures composed of $8 \times 8$ Gabor patches. The task was to detect a square central $4 \times 4$ patch distinguished from the surround by the orientation of the patches, their spatial frequency, or both. Detection accuracy was higher when both cues varied. Performance with the joint cues could not be predicted from any simple linear sum of their independent effects, however, so some nonlinear interaction between the cues was involved. The interaction that Caelli 
and Moraglia hypothesized was one in which "strong codes inhibit weak ones, but otherwise the codes remain largely independent" (p. 683). Evidence for the modulation of luminance boundaries by chromatic cues was reported by Victor, Conte, Purpura, and Katz (1995), using measures of the visual evoked potentials produced by various kinds of textured patterns. They concluded that "when luminance signals are absent, chromatic signals alone fail to support form processing. However, when luminance signals are present, chromatic and luminance signals interact strongly in form processing"'(p. 105). Further evidence for modulation of luminance texture cues by hue comes from implicit effects of hue in two achromatopsic patients. Color cues, of which they report no awareness and which they cannot use as a basis for response selection, nevertheless facilitate the detection of luminance texture boundaries with which they are coincident (Troscianko et al., 1996). A simple interpretation of these implicit influences is that the streams computing color continue to operate up to a level where they modulate the luminance streams, but with their own feedfor- ward outputs no longer functioning normally. This is therefore a dramatic demonstration of the effects of one variable on the transmission of information specifically about another from which it remains distinct. The following section provides a formal analysis of this kind of interaction.

\section{IMPLICATIONS OF ADDITIVE AND MODULATORY INTERACTIONS FOR MEASURES OF TRANSMITTED INFORMATION}

There is a long and strong tradition of using information theory to analyze situations with multiple cues (e.g., Attneave, 1954; Barlow, 1961; Garner, 1962, 1974). We use it here as a way of distinguishing between additive and modulatory interactions. Three binary variables were involved in the following experiments: the orientation (horizontal or vertical) of a texture boundary specified by a contrast in the orientation of the elements, the orientation of a texture boundary specified by a contrast in the
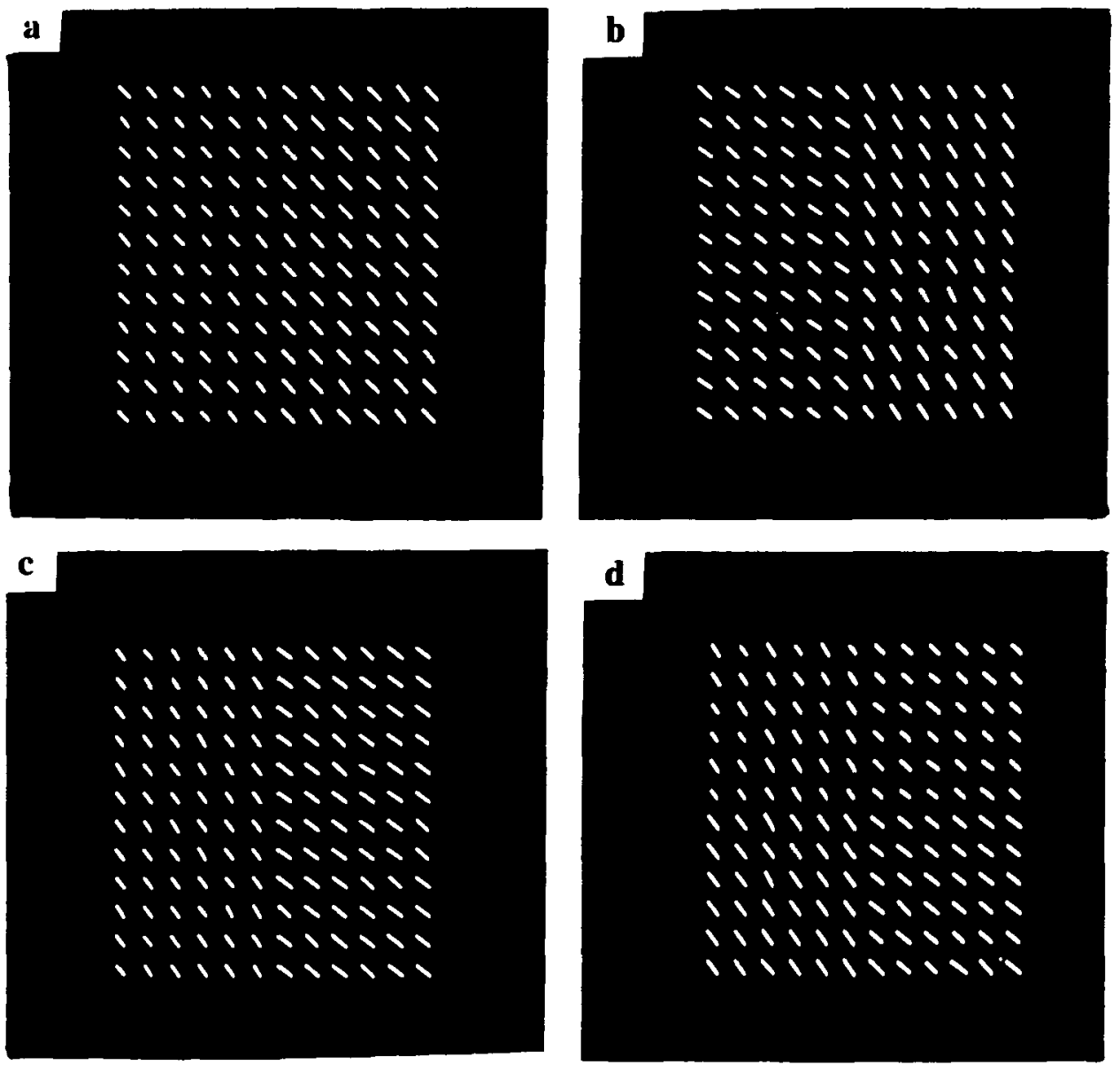

Figure 1. Examples of the texture arrays used. (a) A vertical boundary formed by a length cue. (b) A vertical boundary formed by an orientation cue. (c) A vertical boundary formed by coincident length and orientation cues. (d) A vertical boundary formed by an orientation cue and a horizontal boundary formed by a length cue. The length cue is 1.5 pixels, and the orientation cue is $15^{\circ}$. 
length of the elements, and the orientation specified by the observer's response. Figure 1 shows examples of the texture arrays used. The orientation of the boundary formed by one of the texture cues is specified as the target, and the other is then the nontarget. We will denote the target variable by $T$, the nontarget by $N$, and the response by $X$. The length and orientation texture cues will be referred to as $L$ and $O$, respectively.

For each experiment reported below, $d^{\prime}$ measures for each cue in each condition were calculated in the usual way from the marginal frequency tables, making the usual assumptions of normal distributions with equal variance. Measures based on signal detection theory and those based on information theory are related but are not identical, because information transmitted varies with both $d^{\prime}$ and bias. For a given level of bias, increases in $d^{\prime}$ imply increases in the amount of information transmitted. Changes in response bias alone can also produce changes in the amount of information transmitted, however, with more biased observers transmitting less information. In the limit, observers who are so biased that they only ever produce one of the possible responses transmit no information. Here, we focus on measures of how much information is transmitted by the responses about each cue specifically, as well as on the information transmitted about both cues together. Measures of transmitted information have several advantages. They quantify what can be inferred about the inputs from the output signals produced. They can easily be applied to situations in which there are multiple partially correlated cues. They require few assumptions other than that probability distributions can be estimated from response frequency distributions. Finally, and of particular importance to the aims of this investigation, it has been shown formally how they can be used to distinguish additive from modulatory interactions (Smyth et al., 1996).

Measures of transmitted information were therefore used to analyze the results reported below, so we first outline their basic properties. For a detailed formal presentation of these measures and an account of how they relate to the particular paradigm studied in this paper, see Smyth et al. (1996). The information in the probability distribution of a single variable is a measure of its uncertainty (i.e., how much it varies). A binary variable contains most information when each of its possible values occurs with a probability of .5 . The mutual information between two variables [e.g., $I(X ; T), I(X ; N), I(T ; N)]$ is a measure of how much observing one reduces uncertainty about the other. It is therefore a measure of the association between variables. $I(X ; T)$ is a measure of the amount of information transmitted by the response $X$ about the target $T$. $I(T ; N)$ is a measure of the degree of association between the target and nontarget cues. For two binary variables with individual values that occur with a probability of .5, the mutual information is at a minimum of zero when they are unrelated (i.e., when the probability that they take the same value is .5), and the total information in both is then 2 bits. The mutual information is at a maximum of
1 bit when the probability that they take the same value is either zero or 1 , and the total information in both is then only 1 bit. When the probability that they take the same value is either 1 or .9 , then the mutual information is 0.5 bits, and the total information in both is 1.5 bits. Mutual information thus has a highly nonlinear relation to the probability that two variables take the same value. An improvement in response accuracy from $90 \%$ to $100 \%$ increases the amount of information transmitted far more than does an increase from $50 \%$ to $60 \%$.

We now explain measures of conditional mutual information and their relevance to the analysis of transmitted information in cases with multiple partially correlated cues. Conditional mutual information [e.g., $I(X ; T \mid N)$ ], applies to cases with three or more variables and is a measure of how much observing one variable reduces uncertainty about another, given a third or more variables. It is averaged over all values of the given variables. $I(X ; T \mid N)$ therefore quantifies the information that is transmitted specifically about the target, in addition to any that is transmitted about the nontarget, with which it may or may not be correlated. These measures of transmitted information given multiple cues can be calculated directly from the observed response frequencies (Smyth et al., 1996), and they show how to partition the transmitted information between the various inputs and their interactions. The transmitted information can be partitioned into three disjoint components: $I(X ; T \mid N), I(X ; N \mid T)$, and $I(X ; T \mid N)$. They can be pictured as areas of overlap in a Venn-like diagram composed of the three sets formed by the information contained within each of the three variables (see Figure 4 in Phillips \& Singer, 1997). $I(X ; T \mid N)$ is the information transmitted specifically about the target, in addition to any information transmitted about the nontarget. If the two cues are perfectly correlated, then it will necessarily be zero (i.e., their Venn representations overlap completely). It will be equal to $I(X ; T)$ if the two cues are independent (i.e., their Venn representations do not overlap at all). $I(X ; N \mid T)$ is the information transmitted specifically about the nontarget cue, in addition to any information transmitted about the target cue. If the two cues are perfectly correlated, then this will also be zero. It will be equal to $I(X ; N)$ if the two cues are independent. $I(X ; T \mid N)$ is the information transmitted about the variation that is common to the two cues. If the two cues are independent, then it will be near zero. If the cues are perfectly correlated, then any transmitted information will be composed of this component alone. The total information transmitted about the target, $I(X ; T)$, equals $I(X ; T \mid N)$ plus $I(X ; T \mid N)$ (i.e., the information transmitted that is specific to the target plus the information that is shared with the nontarget). Effects on accuracy as measured by percent correct may therefore be due to effects on either or both of these components.

The implications of three possible forms of interaction for these measures were calculated analytically by Smyth et al. (1996). The three forms of interaction studied were the following: 
1. a simple additive interaction

$$
A=t+n,
$$

where $t$ is the strength of the input specifying the target cue, $n$ is the strength of the input specifying the nontarget cue, and $A$ is the combined strength;

2. a modulatory interaction

$$
A=0.5 t[1+\exp (t n)]
$$

3. a modulatory plus an additive interaction

$$
A=0.5 t[1+\exp (t n)]+n .
$$

Response probabilities were computed by transforming $A$ using the usual logistic function, and the information measures were then calculated directly from those response probabilities.

These three forms of interaction were chosen as representative examples of additive combination, contextual modulation, and both combined. The additive interaction could be enhanced by including parameters that weight the contribution of each cue, as proposed by Landy (1993), but that would make no difference to the contrast with modulatory interactions being studied here. The modulatory interaction given in Equations 2 and 3 above was chosen on both computational and physiological grounds (Kay, Floreano, \& Phillips, 1998; Phillips, Kay, \& Smith, 1995; Phillips \& Singer, 1997) and has effects similar to those produced by the reentrant connections in large physiologically realistic models of the visual system (Tononi et al., 1992). This form of modulation is also similar to that for which there is evidence from functional MR imaging of activity in the visual cortex (Friston, Ungerleider, Jezzard, \& Turner, 1995) and depends on attention (Friston et al., 1997). We therefore use it as an example of modulation, but we do not assume it to be the only form possible. Our emphasis here is not on those properties that distinguish it from other forms of modulation but on those that justify considering it to be a form of modulation and that distinguish it from additive combination.

The properties that distinguish additive and modulatory interactions are shown in Figure 2, which gives the values of the information theory measures as calculated analytically for cases in which a target cue is presented at a range of strengths. The dashed curves show the cases in which the nontarget cue has no effect. The solid curves show the cases in which there is an effect of a nontarget cue of fixed strength that agrees with the target cue on $89 \%$ of trials. The maximum possible value for each of the three components of transmitted information is then 0.5 bits approximately. A priori, one might intuitively suppose that, in all cases, any information that is transmitted specifically about the target must be independent of the nontarget, and vice versa. Figure 2 shows that this is not the case.

The top row of Figure 2 shows the effect of a nontarget cue that is additively combined with the target cue. The effect of presenting the nontarget can be seen by comparing outcomes when the nontarget strength is 2 with that when it is zero, which is equivalent to not presenting the cue. Presenting the nontarget reduces the information transmitted specifically about the target, $I(X ; T \mid N)$. However, it has little effect on the rate at which $I(X ; T \mid N)$ increases with increases in the strength of the target (i.e., the nontarget does not affect target information transmission gain). The information transmitted specifically about the nontarget cue, $I(X ; N \mid T)$, is maximal when the target cue is too weak to have any effect and decreases monotonically as the strength of the target cue increases. These effects can be understood intuitively in terms of competition between the cues for output channel capacity. The greater the specific effect of one cue, the less that of the other. (From this perspective, response bias can be seen as an uncorrelated nontarget variable that reduces target information transmission by combining additively with it to determine performance. The reduction will be small unless the bias is large.)

The mutually supportive aspect of an additive interaction is shown by an increase in $I(X ; T \mid N)$ when both input cues are used. However, the gain of this measure, which for simplicity is not shown in Figure 2, is little affected by an additive interaction. These effects show why the target cue is only nominally the target when multiple cues are additively combined to determine response. The response transmits information about any specific cue only if we know the others. Nothing can be inferred from the responses alone about any cue specifically. This is compatible with interpreting additive combination as computing a new higher order variable.

The middle row of Figure 2 shows the modulatory interaction. In contrast to an additive interaction, there are values of target strength at which presenting the nontarget increases the information transmitted specifically about the target. A second contrast is that it effects the rate at which $I(X ; T \mid N)$ increases with increases in the strength of the target cue. This can be understood intuitively as showing modulation, because the modulator changes the rate at which information transmitted specifically about a target increases with target strength (i.e., it changes information transmission gain). A third contrast is that $I(X ; N \mid T)$ is zero when the target cue is too weak to have any effect and then increases before decreasing monotonically as the strength of the target cue increases. This can be understood intuitively as showing modulation, because the modulator has no effect by itself, but an effect is produced as target strength increases. A fourth contrast is that the rate of change of $I(X ; T \mid N)$ as a function of target strength depends on nontarget strength. These effects show the sense in which the output of a modulatory interaction can be thought of as still transmitting information about the target, rather than about a new higher order variable formed by the interaction. The output transmits information specifically about the target no matter what the context, but it transmits information specifically about the context only for a restricted set of values of the target. Something can be inferred from the output specifically about the target whether or not the 
a)

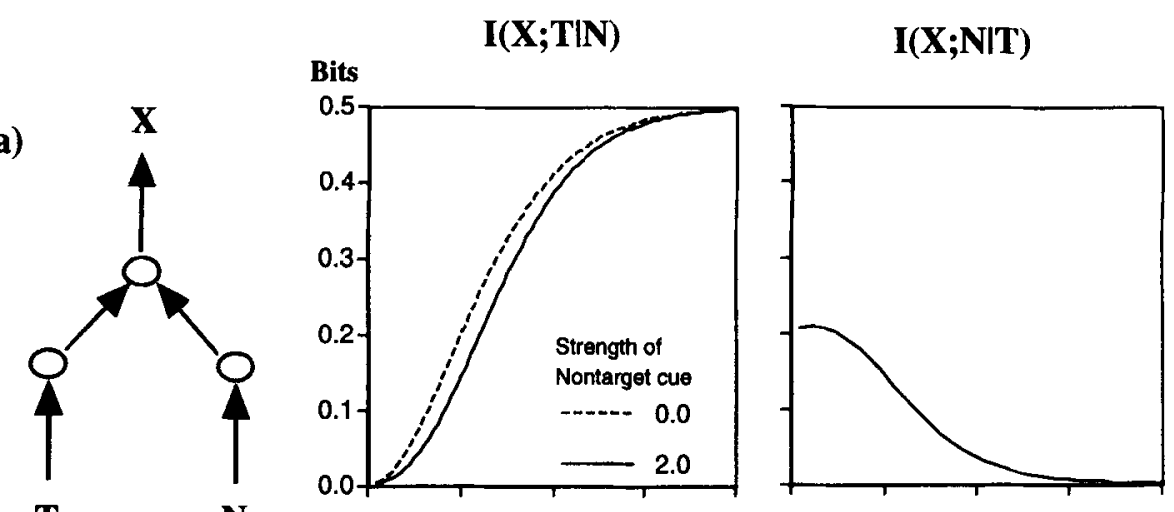

b)
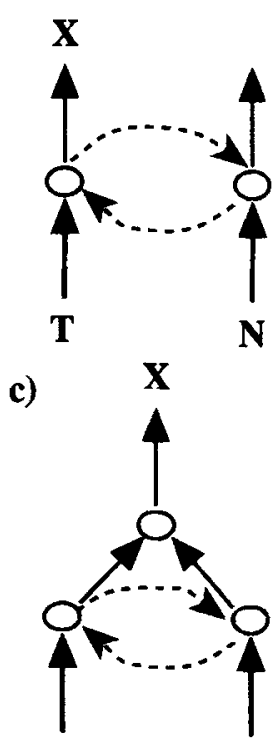

$\begin{array}{cc}\text { Target } \\ \text { cue } & \begin{array}{c}\text { Nontarget } \\ \text { cue }\end{array}\end{array}$
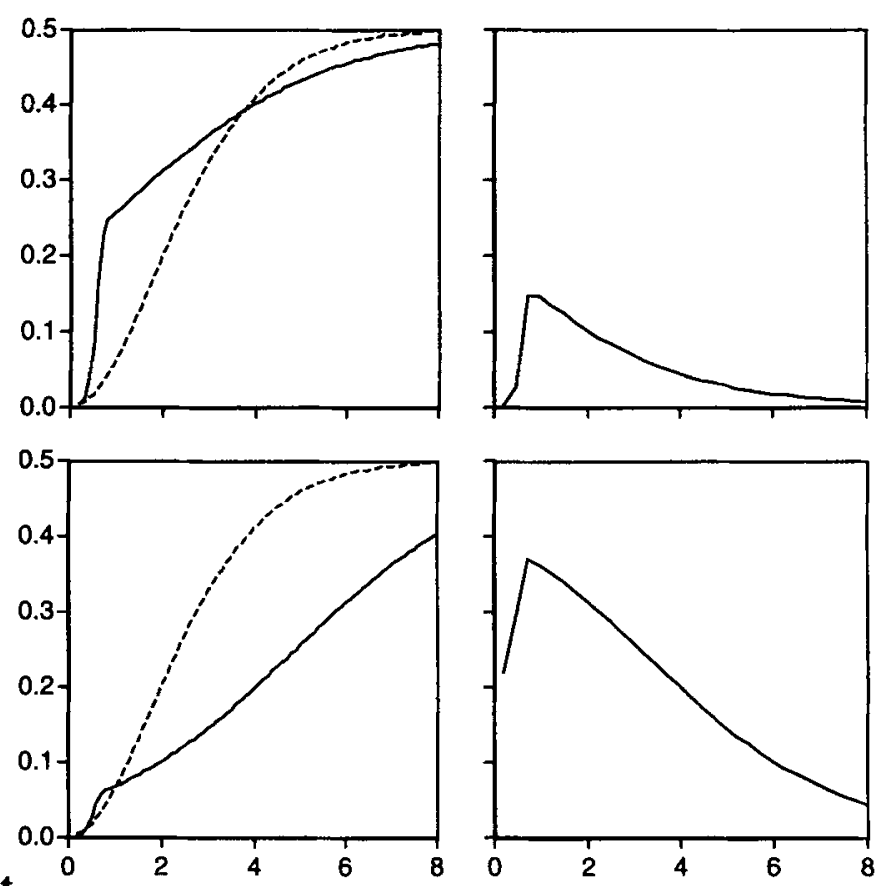

strength of target cue

Figure 2. Possible interactions between cues and their implications for the transmission of information specifically about the target cue, $I(X ; T \mid N)$, and specifically about the nontarget cue, $I(X ; N \mid T)$. All information measures were calculated analytically using the forms of interaction specified in the text, (a) An additive interaction in which input cues are combined to compute a higher order feature. (b) A modulatory interaction (shown by dotted arrows) in which each cue modifies transmission of information about the other. For simplicity, only a single cue is shown in each of the distinct streams of processing, but they can also consist of multiple cues combined in various ways. (c) A modulatory interaction followed by an additive interaction.

context is known, but nothing can be inferred specifically about the context unless the target is known. Modulation can therefore also be understood intuitively in terms of disambiguation. Contextual modulation only has any effect if the target is ambiguous, and its effect is to amplify interpretations of the target for which there is evidence and that fit the context.

The bottom row of Figure 2 shows the effects of modulatory and additive interactions combined. It shows the same distinctive signs of the presence of a modulatory interaction, as in the case of pure modulation just described.
The increased gain of $I(X ; T \mid N)$ at low target strengths is small in Figure 2c, but it is greater if the nontarget cue is weaker than that used here (Smyth et al., 1996).

To analyze the psychophysical data obtained in the experiments reported below, the information measures were calculated directly from the probabilities estimated from the response frequencies in order to see whether the distinctive effects of additive and/or modulatory interaction occur. The issue being addressed here is to distinguish between additive and modulatory interactions in general. Our strategy was therefore to seek psychophysical evi- 
dence relevant to the properties that distinguish them, rather than to fit theoretical functions to the data. General $\operatorname{logLinear}$ multiway frequency analysis (Tabachnick \& Fidell, 1989) was used to test the reliability of the relevant associations in the response frequency tables. This analyzes the significance of conditional associations and interactions, which is appropriate given our focus on conditional mutual information measures.

\section{GENERAL METHOD}

\section{Observers}

Six male observers from the Department of Psychology at the University of Stirling were studied: Two (B.C. and W.P.) were the authors, 2 (E.M., and A.W.) were final-year psychology undergraduates, and 2 (G.L. and P.T.) were postgraduate psychology research students. All reported normal or corrected-to-normal visual acuity. Three of these observers (E.M., W.P., and A.W.) were tested extensively in more than 10 different experiments per subject, with each experiment involving between 400 and 1,000 trials. The observers were aware of the general issues being studied, but they did not know what the outcome of each experiment would be, and this typically remained the case even after they had finished all the trials in an experiment.

\section{Apparatus}

The stimuli were generated on a Viglen Genie 4DX66 computer and displayed on a Viglen Envy 15-in. color monitor with a refresh rate of $72 \mathrm{~Hz}$. The monitor screen measured $25.5 \mathrm{~cm}$ horizontally $\times 19.0 \mathrm{~cm}$ vertically. There were 640 pixels horizontally and 480 pixels vertically. Normal room lighting gave a dark screen luminance of approximately $15 \mathrm{~cd} / \mathrm{m}^{2}$. The maximum brightness produced by the monitor was approximately $120 \mathrm{~cd} / \mathrm{m}^{2}$. The stimuli were viewed binocularly from a distance of approximately $60 \mathrm{~cm}$.

RTs were measured by checking for a keypress during every vertical retrace of the monitor and counting the number of monitor refresh cycles that passed before a keypress was made.

\section{Stimuli}

Stimuli consisted of $12 \times 12$ arrays of white lines on a dark background, the midpoints of the lines lying on a square grid with horizontal and vertical spacings of 20 pixels (Figure 1). Each line could be adjusted in length and orientation. To create each line, a notional infinitesimally thin line of the appropriate length and orientation was blurred by convolution with a 2-D Gaussian function with a standard deviation of 1.5 pixels. This technique enabled the lengths of the lines to be varied with subpixel precision and their orientation to be varied continuously without any visible "staircasing." The brightness of the lines was not varied as their length varied, and, consequently, the total luminous energy of a line covaried with its length. For our purposes, this covariation was not important: Our arguments would not be altered if "length cue" were to be replaced by "length plus luminous energy cue" throughout. Similarly, varying the length and orientation of line elements produces changes in the spatial frequency content of the stimuli. This does not change the issue being studied here, however, because we were concerned with determining whether the information provided by one cue modulates or adds to the information provided by the other, rather than with delineating the detailed spatial frequency content of those cues.

The nominal line length was 10 pixels. The actual line lengths varied from this figure in two ways. First, there was a difference between the two halves of the array of lines in the means of the Gaussian populations from which the line lengths were drawn. This difference in the population mean line length (which might be zero) was a cue by which the observers could potentially segment the array into two halves. A value of the cue of $x$ pixels meant that the population mean line length in one half of the array was $10+x / 2$ and that in the other half of the array was $10-x / 2$. The division of the array could be along either its vertical or horizontal midline. The second source of variation in line length was random variation from line to line. The population standard deviations of the two distributions of line lengths were equal at 0.7 pixels.

The orientations of the lines were selected in an analogous fashion and thus provided a second cue (which might be zero) by which the observer could potentially segment the array into two halves. A value of the cue of $x$ degrees meant that the population mean orientation in one half of the array was $10+x$ and that in the other half of the array was $10-x$. The division of the array by this cue was also along its vertical or horizontal midline. The nominal orientation was $45^{\circ}$ counterclockwise from the vertical, and the standard deviation of orientation was $3^{\circ}$. The division of the stimulus array on the basis of line orientation did not necessarily have the same orientation (horizontal or vertical) as the division based on line length. The probability with which the two cues indicated the same division of the stimulus array was one of the parameters of the sets of stimuli presented in each experiment.

In the first three experiments, in which we tested for modulatory interactions between stimulus cues, we studied cases in which the cues were partially correlated within the experiment. Not only was this more ecologically valid but it reduced the chance that the experiment itself would reduce or remove the interaction for which it was testing. There is evidence that interactions between cues can be modified by training (Polat \& Sagi, 1994), so if no evidence for modulatory interactions is found in experiments using uncorrelated cues, as in most previous experiments, then it could be argued that this may be because the visual system has adapted to the independence between cues experienced during the experiment.

\section{Experimental Design and Procedure}

On each trial, the stimulus was displayed until the observer had made a response or until $1 \mathrm{sec}$ had elapsed, whichever was sooner. After the observers had made their response, an interval of $2 \mathrm{sec}$ elapsed before presentation of the next stimulus.

The observer's task was to decide whether the relevant texture boundary in the stimulus array was horizontal or vertical and to indicate their response using one of two keys on the computer's numeric keypad. The relevant texture boundary was specified in terms of that formed either by the length cue or by the orientation cue. For unpracticed observers, the distinction was made clear by showing examples in which the boundary formed by each cue was easily seen. The observers were instructed to make their response as quickly as they could, but not to sacrifice accuracy for speed. For each experiment, the observers typically did several hundred trials, broken into blocks of a few hundred. An interval of at least $1 \mathrm{~h}$ was left between successive blocks of trials.

As in the studies of Nothdurft (1992), the observers' responses in these experiments seemed to be based on a global impression, rather than on a detailed analysis of individual elements. This is in keeping with the short display time used and with the essentially statistical nature of the task.

\section{EXPERIMENT 1 Effects of a Strong Orientation Boundary on the Transmission of Information About a Target Length Boundary}

The goal of Experiment 1 was to determine whether an orientation texture boundary has any effect on the trans- 
mission of information about a target length texture boundary, and, if so, whether it increases or decreases the information transmitted specifically about the target. If the observer's response is based only on a generalized texture boundary map that does not distinguish the cues used, then the orientation boundary will have large effects, improving performance when coincident and worsening it when orthogonal. If only additive interactions occur, then presenting the nontarget cue will decrease the information transmitted specifically about the target. If it increases that information, however, then it will provide evidence for modulatory interactions. It is also possible that the orientation boundary will have no effects, given highly practiced observers who may be able to ignore the nontarget cue. Yet another possibility is that the nontarget cue will act only as some kind of mask, worsening perfor- mance whenever it is strong enough to have any effect, as suggested by Caelli and Moraglia (1985).

\section{Method}

Two practiced observers were used (W.P. and A.W.). The texture patterns and general method were as described above. The observers were instructed to report the orientation of the texture boundary formed by the length cue. Two levels of this cue were used: 0.5 and 1.5 pixels, where this gives the mean difference in the length of the line elements between the two halves of the array separated by the length texture boundary. Two values for the orientation cue were used: $0^{\circ}$ and $15^{\circ}$, where this gives the difference in the orientation of the line elements between the two halves of the array separated by the orientation texture boundary, as described in the General Method section. This gives four different conditions, in two of which only the target cue was present and in two of which there was also a nontarget cue. The four different conditions occurred equally often and randomly across trials. The probability that the
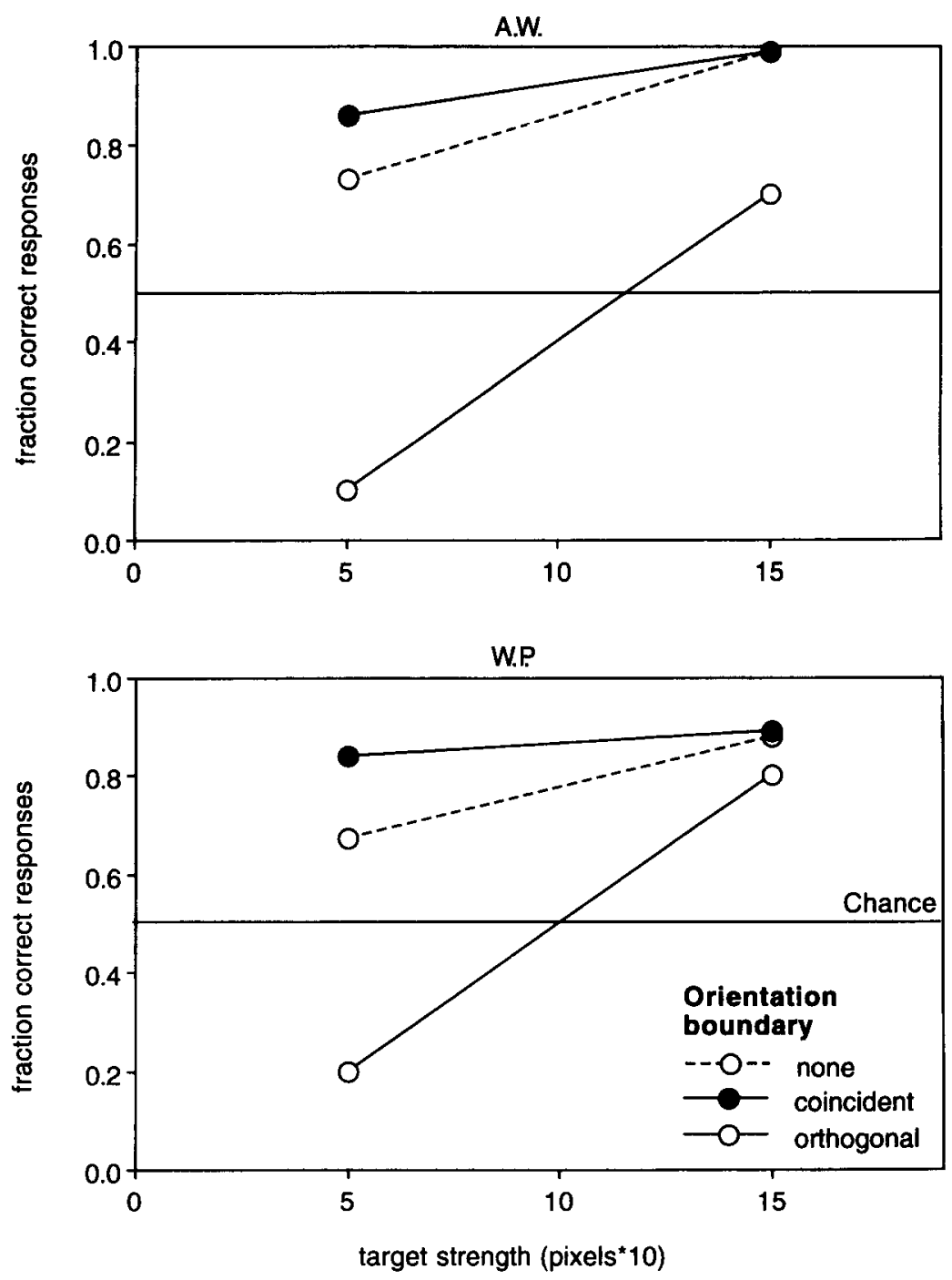

Figure 3. Response accuracy in Experiment 1 for the 2 observers, A.W. and W.P., shown as a function of the strength of the target cue and its relation to the nontarget cue. 
two cues were coincident was .9. At this value, $I(L ; O)=I(L \mid O)=$ $I(O \mid L)=0.5$ approximately. Thus, the maximum possible value for each of $I(X ; L ; O), I(X ; L \mid O)$, and $I(X ; O \mid L)$ is also approximately 0.5 . The observers performed 22 trials in each condition in each of five blocks of trials, requiring about $10 \mathrm{~min}$ per block and providing 110 trials for each condition for each observer.

\section{Results and Discussion}

The percentages of responses that correctly matched the target cue in the presence of coincident, orthogonal, and no nontarget cue are shown in Figure 3. Accuracy was improved by a coincident boundary and was worsened by an orthogonal one. The effect of the nontarget cue was clearest at the lower target strength. However, simply noting this effect of the nontarget cue on accuracy does not distinguish additive and modulatory effects. Evidence on this can be obtained by dividing the transmitted information into its three components: that specific to the target, $I(X ; L \mid O)$; that specific to the nontarget, $I(X ; O \mid L)$; and that common to both, $I(X ; L ; O)$. When the nontarget boundary was made undetectable by setting the orientation difference to zero, the three components were $0.025,0.006,0.066$, respectively, for Observer W.P. and were $0.048,0.001$, and 0.108 , respectively, for Observer A.W. When the nontarget boundary was $15^{\circ}$, the three components were $0.001,0.115$, and 0.243 , respectively, for Observer W.P. and were 0.010 , 0.175 , and 0.250 , respectively, for Observer A.W. The maximum value for each of these components is about 0.5 bits. All measures given are for the lower target strength. The observers were therefore primarily responding to the information common to the two cues. As expected of an additive interaction, presentation of a nontarget cue decreased the amount of information transmitted specifically about the target cue, so this provides no evidence for a modulatory interaction. The amount of information transmitted specifically about the nontarget cue was necessarily near zero when that cue was not presented and increased substantially when it was presented, which is again as expected of an additive interaction.

To test the reliability of the observed effects a four-way frequency analysis was performed to develop a hierarchical log linear model of response frequencies. The variables used were response (correct or not), length cue $(0.5$ or 1.5 pixels), orientation cue (coincident, orthogonal, or none), and observer (W.P. or A.W.). The simplest model producing a satisfactory fit [deviance $(12)=14.15, p>$ .2] to the observed frequencies included the two-way association between accuracy and each of the other three variables, a three-way association between accuracy, length, and observer, and no other higher order associations. The association between accuracy and the nontarget cue was highly significant [deviance $(14)=63.7, p<$ $.001]$ and did not interact with observer or with target strength. Separate comparisons of performance at the weaker length cue ( 5 pixels) showed that accuracy was significantly higher with a coincident orientation cue than with none for each observer [W.P., deviance $(1)=8.051$, $p<.005$; A.W., deviance $(1)=5.694, p<.025]$. They also showed that accuracy was significantly lower with an orthogonal orientation cue than with none for each observer [W.P., deviance $(1)=8.619, p<.005$; A.W., deviance $(1)=15.93, p<.001]$.

RTs for correct response with coincident, orthogonal, and no nontarget boundary reflected the accuracy measures (Figure 4). Correct responses were faster with a coincident nontarget boundary and was slower with an orthogonal one.

The results show that both cues affect performance even when only one is specified as the target and when observers are highly practiced. They also show that the cues do not interact only by masking each other. They are evidence for a generalized texture boundary map that combines texture gradients from different cues without distinguishing them, as shown in Figures $2 a$ and $2 c$.

Evidence that observers do not reliably distinguish different texture boundary cues does not imply that they have no ability to do so, however, and there is a suggestion in these results that they may have some such ability. This is because responses more often matched the target than the nontarget when both cues were strong. However, the target may have been stronger than the nontarget in this particular case, so this issue was studied further in Experiments 4 and 5 .

Evidence for summative interactions is not evidence against modulatory interactions; it is possible that both occur, as shown in Figure 2c. Experiment 2 therefore continued the search for signs of modulatory interaction, but using a weaker nontarget boundary, because that makes it easier to find such signs in the case in which the nontarget also makes an additive contribution.

\section{EXPERIMENT 2 \\ Effects of a Weak Orientation Boundary on the Gain of Information Transmission About a Target Length Boundary}

Experiment 2 was designed to find out whether there are effects of a weak orientation boundary on the gain of information transmission about a target length boundary. Three values for the length cue were chosen so as to measure gain at low target strengths, because that is where modulation affects target information transmission gain, as shown in Figure 2. Single-unit studies of facilitation in V1 shows that it is more likely to occur at low target strengths (Polat et al., 1998). If an analogous facilitation occurs between texture boundary cues, then it is at low target strengths that gain should be most enhanced.

A weaker nontarget cue was used in Experiment 2 than in Experiment 1. This is because Experiment 1 gave evidence of an additive interaction between the cues, in which case a strong nontarget can play the dominant role in determining response and thus obscure any modulatory effect that it might have on the processing of the target. 

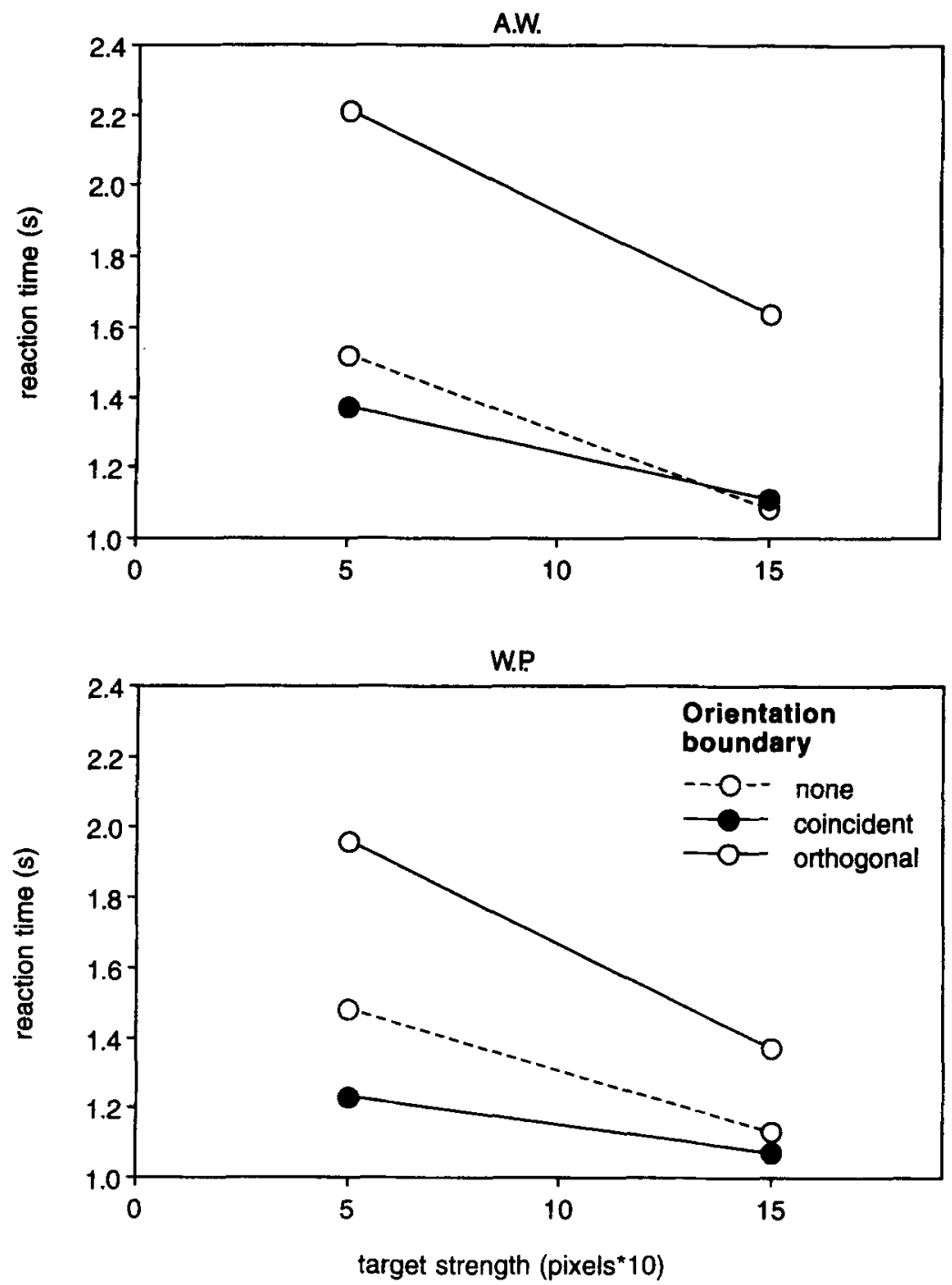

Figure 4. Reaction times (in seconds) of correct responses in Experiment 1 for the 2 observers, A.W. and W.P., shown as a function of the strength of the target cue and its relation to the nontarget cue.

\section{Method}

The same 2 practiced observers (W.P. and A.W.) were used as in Experiment 1. The texture patterns and general method were as described above. The observers were instructed to report the orientation of the texture boundary formed by the length cue. Three levels of this cue were used: $0.3,0.6$, and 0.9 pixels, where this gives the mean difference in the length of the line elements between the two halves of the array separated by the length texture boundary. An orientation cue of $5^{\circ}$ was present on half of the trials at random. This gives six different conditions, in three of which only the target cue was present and in three of which there was also a nontarget cue. The six different conditions occurred randomly and with equal probability across trials. The probability that the two cues were coincident was .9 . The observers performed 22 trials in each condition in each of 10 blocks of trials, requiring about 15 min per block and providing 220 trials for each condition and for each observer.

\section{Results and Discussion}

The percentage of responses that correctly matched the target cue in the presence of coincident, orthogonal, and no nontarget cue showed the same effects as in Experiment 1 . Accuracy was improved by a coincident boundary and was worsened by an orthogonal one. The nontarget did not affect the gain of sensitivity to the target, however. Estimates of $d^{\prime}$ for each of the three nontarget cue conditions at each of the three target strengths gave higher values for $d^{\prime}$ with a coincident nontarget cue, and lower values with an orthogonal nontarget cue when compared to no nontarget cue, but the rate at which $d^{\prime}$ increased with increases in target strength was approximately the same in all three conditions. The effects of presenting the non- 

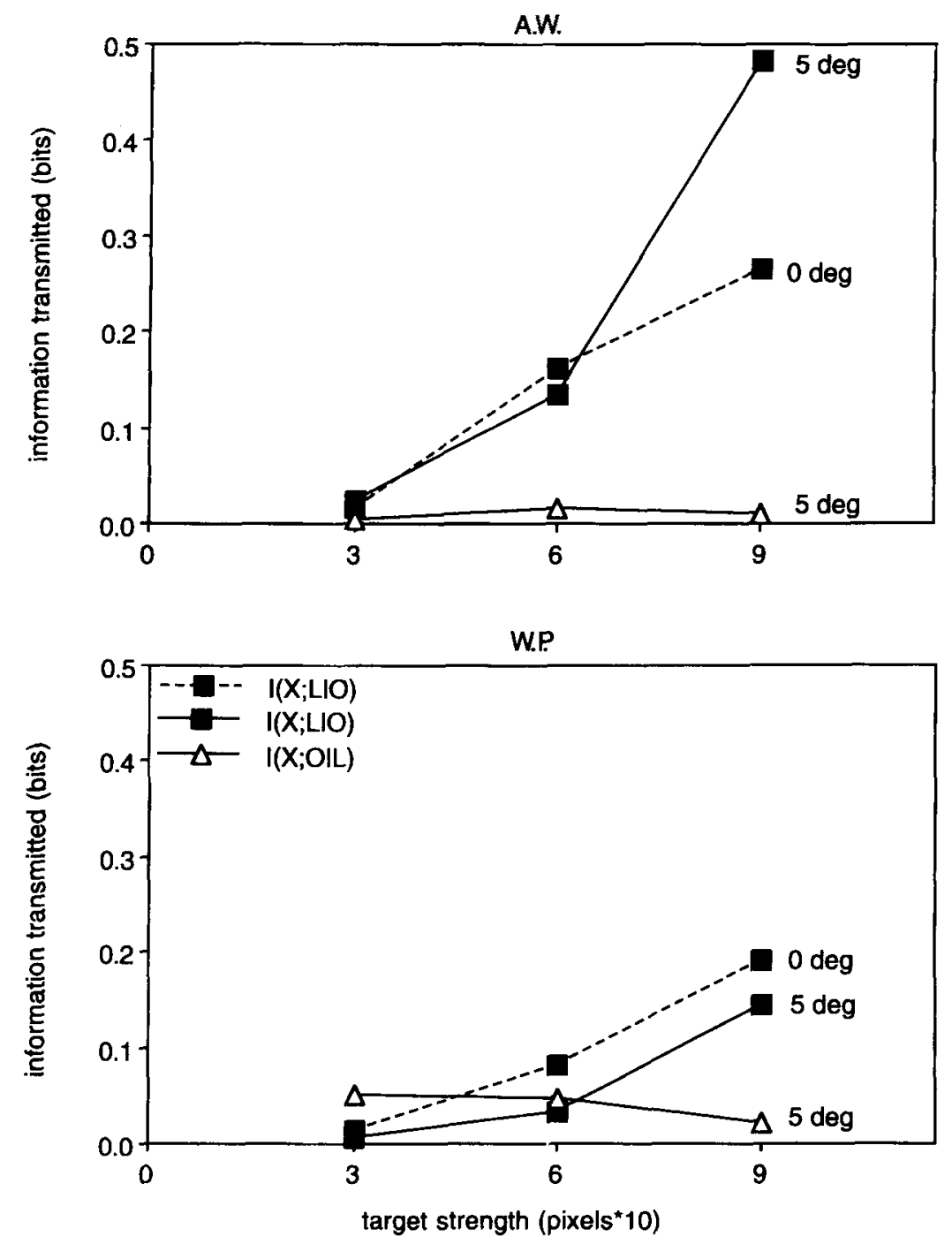

Figure 5. Measures of the information transmitted in Experiment 2 for the 2 observers, $A . W$. and W.P., shown as a function of the strength of the target cue with the strength of the nontarget cue as a parameter. $I(X ; L \mid O)$ shows the amount of information transmitted specifically about the target length boundary. $I(X ; O \mid L)$ shows the amount of information transmitted specifically about the nontarget orientation boundary.

target cue on the conditional mutual information measures $I(X ; L \mid O)$ and $I(X ; O \mid L)$ were calculated and are shown in Figure 5. Presenting a weak orientation boundary has no clear effect on the gain of information transmission about the length boundary. Thus, this seems to provide no evidence for modulatory interactions. Furthermore, if the effect of the orientation boundary was modulatory, then the information transmitted specifically about it, $I(X ; O \mid L)$, would increase with increases in target strength. Figure 5 does not show any such effect.

To test the significance of the various two-way and higher order interactions, a four-way frequency analysis was performed to develop a hierarchical log linear model of response frequencies. The variables used were response (correct or not), length cue $(3,6$, or 9 pixels), orientation cue (coincident, orthogonal, or none), and observer (W.P. or A.W.). The simplest model producing a satisfactory fit [deviance $(24)=26.96, p>.25$ ] to the observed frequencies included the two-way association between accuracy and each of the other three variables, and no other two-way or any higher order associations. The association between accuracy and length cue was highly significant [deviance $(26)=201.4, p<.001$ ] . The association between accuracy and nontarget cue was also highly significant [deviance $(26)=58.01, p<.001$ ] . This shows that, though weak, the nontarget cue still had a highly significant effect. There was no evidence for an increase in the effect of the nontarget cue on accuracy with increases in 
target strength, however, as shown by the good fit of a model without any three-way interactions. Thus, this analysis found no evidence for modulatory interactions.

As in Experiment 1, RT measures reflected the accuracy measures. Correct responses were faster with a coincident nontarget boundary and were slower with an orthogonal one. Again, they fail to provide any evidence for modulatory effects of the nontarget cue, in that the effects of the nontarget cue on RT did not increase with increases in target cue strength, and effects of the nontarget cue on RT occurred together with effects on accuracy.

These results provide no evidence that a weak orientation boundary affects the gain of information transmission about a target length boundary. Experiment 1 found no evidence for modulatory effects of a strong orientation boundary. Failure to find evidence for modulatory effects of orientation cues on the processing of length cues in Experiments 1 and 2 does not show that it does not occur under other conditions, but many other variants of this paradigm were run with Observers W.P., A.W., and E.M.S., and no reliable evidence for modulatory effects was found in any of them. Our working hypothesis is therefore that an orientation cue does not modulate the transmission of information about a coincident length cue, which is processed independently of the orientation cue before being combined with it in a generalized texture boundary map.

\section{EXPERIMENT 3 \\ Effects of a Length Boundary on the Gain of Information Transmission About a Target Orientation Boundary}

Experiments 1 and 2 show that an orientation boundary affected judgments of a length boundary but did not affect the gain of information transmission about that boundary. Experiment 3 examined the effects of a length boundary on judgments of a target orientation boundary. Any effects due to additive combination will be reciprocal. Effects due to modulatory interaction need not be.

In addition, Experiment 3 tested for suppressive effects and facilitatory effects of modulation. Both are produced by the modulatory interaction shown in Figure 2, but they need not occur together. In Experiment 3, therefore, more observations were taken at higher levels of target strength, where there is more room for any suppressive component of modulation to be expressed. If suppression occurs, then the slope of the upper part of the psychometric function will be less with than without a nontarget cue.

\section{Method}

Four observers were studied: One (W.P.) was highly practiced at this task, and 3 (B.C., G.L., and P.T.) had not been used as observers prior to this experiment. The texture patterns and general method were as described above. The observers were instructed to report the orientation of the texture boundary formed by the orientation cue. Five levels of this cue were used: $0^{\circ}, 5^{\circ}, 10^{\circ}, 15^{\circ}$, and $20^{\circ}$. Two values for the length cue were used: 0 (i.e. no length cue) and 1.0 pixels. This gives 10 different conditions, including one in which the task was impossible because both cues were zero. The observers were informed that a few trials would be impossible, but they were instructed to guess anyway. This had the advantage of helping the observers feel comfortable with having to guess on some trials. The 10 different conditions occurred randomly and with equal probability across trials. The probability of the two cues being coincident was .7. The observers performed 20 trials in each condition in each of five blocks of trials, requiring about 20 min per block and providing 100 trials for each condition and for each observer. The observers performed 60 practice trials prior to the first block of experimental trials.

\section{Results and Discussion}

The percentage of responses that correctly matched the target cue in the presence of coincident, orthogonal, and no nontarget cue showed the same effects as in Experiments 1 and 2. Accuracy was improved by a coincident boundary and worsened by an orthogonal one. Figure $6 \mathrm{a}$ shows means over all 4 observers combined, since all show essentially the same effects, and differences between observers did not approach significance in the multiway frequency analysis reported below. Estimates of $d^{\prime}$ for each of the three nontarget cue conditions at each target strength gave higher values for $d^{\prime}$ with a coincident nontarget cue and lower values with an orthogonal nontarget cue when compared with no nontarget cue. However, the rate at which $d^{\prime}$ increased with increases in target strength was approximately the same in all three conditions. The information transmitted specifically about the target, $I(X ; O \mid L)$, and about the nontarget, $I(X ; L \mid O)$, is shown in Figure $6 \mathrm{~b}$. Presenting a length boundary did not increase the rate at which information specific to the target orientation boundary increased from zero at low target strengths, nor did it decrease it at higher target strengths. Thus, this provides no evidence for modulatory interactions. Furthermore, if the effect of the nontarget had been modulatory, then the information transmitted specifically about it, $I(X ; L \mid O)$, would increase with increases in target strength. As in Experiment 2 , there was no evidence for this.

A four-way frequency analysis was again performed to develop a hierarchical log linear model of the response frequencies. The variables used were response (correct or not), orientation cue $\left(0^{\circ}, 5^{\circ}, 10^{\circ}, 15^{\circ}, 20^{\circ}\right)$, length cue (coincident, orthogonal, or none), and observer (W.P., B.C., G.L., P.T.). The simplest model producing a satisfactory fit [deviance $(103)=79.63, p>.25]$ to the observed frequencies included only the first-order terms and the twoway associations between accuracy and target length cue and between accuracy and the nontarget orientation cue. The association between accuracy and the strength of the target orientation cue was highly significant [deviance $(107)=858.0, p<.001]$. The association between accuracy and the nontarget length cue condition was also highly significant [deviance $(105)=152.0, p<.001$ ]. This shows that, though weak, the nontarget cue still had a highly significant effect. The three-way interaction between accuracy, target cue, and nontarget cue did not approach significance. Therefore, these results do not show any effect of the nontarget cue on target information transmission gain.

Correct responses were faster with a coincident nontarget boundary than with an orthogonal one. Again, they fail to provide any evidence for modulatory effects of the 

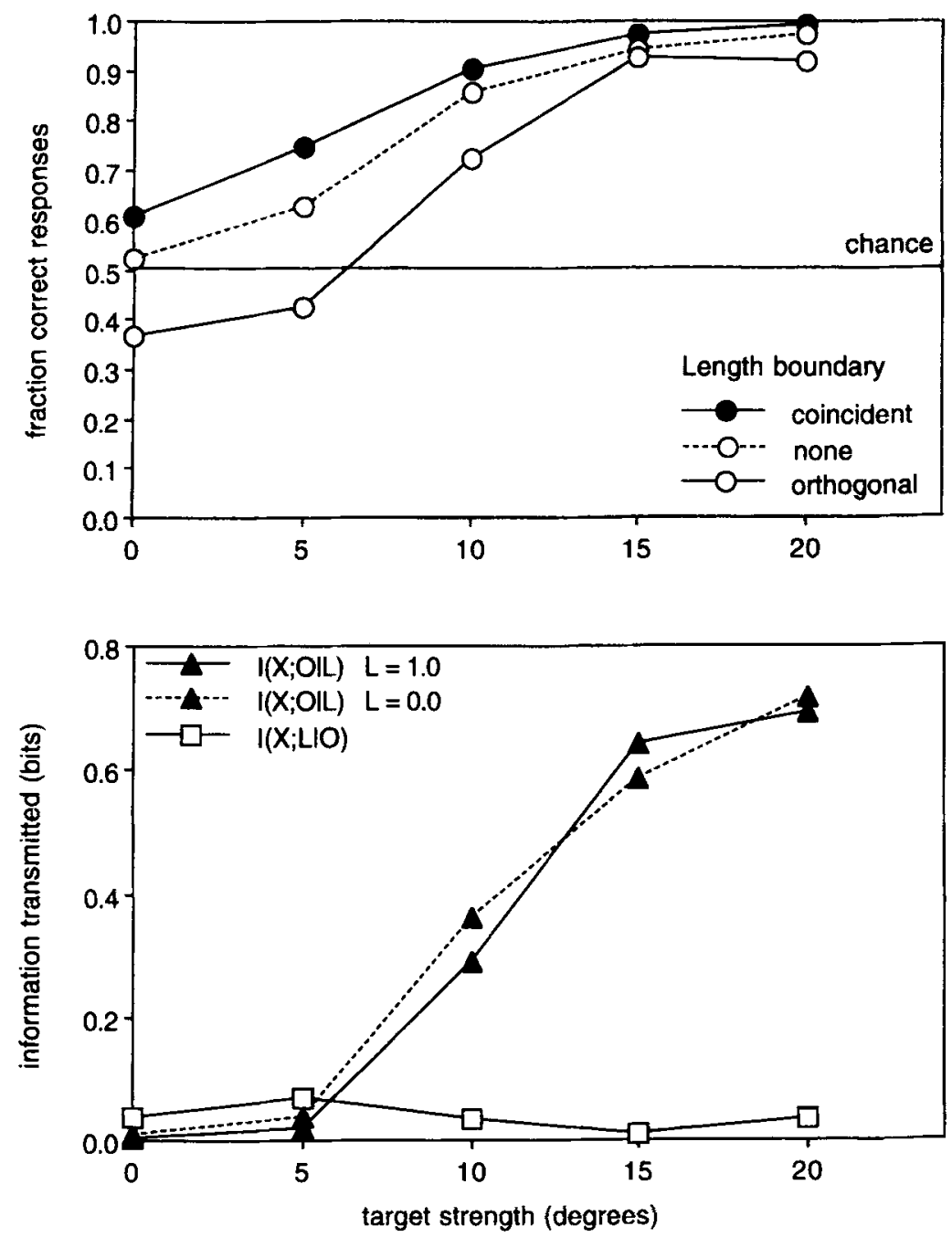

Figure 6. Response accuracy and information transmitted in Experiment 3 averaged across all 4 observers, shown as a function of the strength of the target orientation cue. The upper panel is parameterized by the condition of the nontarget length cue. In the lower panel, $I(X ; O \mid L)$ shows the amount of information transmitted specifically about the target orientation boundary, and $I(X ; L \mid O)$ shows the amount of information transmitted specifically about the nontarget length boundary.

nontarget cue, in that the effects of the nontarget cue on RT did not increase with increases in target cue strength, and effects of the nontarget cue on RT occurred together with effects on accuracy.

The implication of these results seems clear. Length texture boundaries sum with orientation texture boundaries, but they do not affect their information transmission gain. The interactions between these cues were reciprocal, as expected of additive interactions. The results provide no evidence either for facilitatory modulation of weak orientation cues or for suppressive modulation of strong orientation cues. The absence of any significant differences between observers suggests that these effects do not depend greatly on the amount of practice at the task, because the task was highly familiar to 1 of the observers,
W.P., who had performed more than 10,000 trials in various versions of the paradigm prior to this experiment, whereas the task was new to the other 3 observers.

\section{EXPERIMENT 4 \\ Effects of Task Context on the Contribution of Different Texture Boundary Cues to Response}

The results of Experiments 1-3 show that both cues influenced response even though only one was specified as the target. Experiment 4 studied whether the contribution of each cue to response is fixed, as in the simple summative model shown in Figure 2a, or whether it depends on the task. To study this issue, identical stimuli were used with two different tasks: one in which the texture bound- 
ary formed by the length cue was specified as the target, and one in which that formed by the orientation cue was specified as the target. The strength of the two cues varied randomly and independently from trial to trial, as did the orientation of the boundaries that they formed. The orientations of the boundaries formed by the two cues were not correlated within the experimental paradigm. Since this experiment was not seeking modulatory interactions between stimulus cues, the possibility of weakening any such interactions by decorrelating the cues within the experiment was no longer a concern. Performance was also studied with each cue at each strength presented alone.

In the Discussion section of Experiment 1, it was noted that responses more often matched the target than the nontarget when both cues were strong and that this may have been because the target cue happened to be even stronger than the nontarget cue in that case. If so, then responses will continue to match the stronger cue even when the other is specified as the target. If response is more likely to match a cue when it is specified as the target, however, then this will be evidence that the two cues can, to some extent, be distinguished and that their effects depend on task context.

\section{Method}

Two practiced observers (W.P. and A.W.) were used. The texture patterns and general method were as described above. The texture boundary formed by the length cue was the target in half of the blocks of trials, and the texture boundary formed by the orientation cue was the target in the other half. Two values of the length cue were used: 0.5 and 1.5 pixels. Two values for the orientation cue were used: $5^{\circ}$ and $15^{\circ}$. This provided a weak value and a strong value for each cue, so that any task effects would be found even if they applied only to strong or weak cues. Trials were also run in which only one cue was present, it being the target. This gives four conditions in which the two cues were present and the length cue was the target, another four conditions differing only in that the orientation cue was the target, and four with only a target cue. A full block of trials consisted of four parts: one cue alone, and then as target when presented with the other cue, then the other cue alone, then as target when presented with the other cue. The order in which the cues were specified to be the target was alternated across blocks. The probability that the two cues were coincident was .5. The observers performed 20 trials in each condition in each of four full blocks of trials, requiring about $30 \mathrm{~min}$ per block and providing 80 trials for each of the 12 conditions for each observer.

\section{Results and Discussion}

For Observer W.P., response accuracy in trials with a single cue was $67.5 \%$ and $99 \%$ for a $5^{\circ}$ and $15^{\circ}$ orientation cue, respectively, and $65 \%$ and $94 \%$ for a $5-$ and a 15-pixel-length cue, respectively. The equivalent scores for Observer A.W. were $62.5 \%$ and $96.25 \%$, and $68.75 \%$ and $98.75 \%$. Effects on response accuracy in trials with two cues were as follows. First, they confirm that the roles of the two cues as target and nontarget can be reversed and still produce essentially the same results. Second, if practiced observers can completely ignore the nontarget cue, then the percentages of correct response would not depend on whether the nontarget cue was present, coincident, or orthogonal. They clearly did, however; so this possibility can be rejected. Third, if the two cues affect response only through a generalized texture boundary map that is automatically computed and that does not distinguish different texture boundary cues, as shown in Figure $2 a$, then responses should not be more likely to match a particular cue when that cue is specified as the target. However, the responses to orthogonal cues did depend on which was specified as the target. One way to see this is to look at the effect of task instructions on response to orthogonal cues. Given two strong orthogonal cues, for example, the responses of Observer W.P. matched the length cue on $92.5 \%$ of trials when it was the target, but only on $25 \%$ of the trials when it was not the target. The equivalent scores for Observer A.W. were $97.5 \%$ and $25 \%$. The possibility that responses are determined by an automatically computed generalized map can therefore also be rejected. Another way to see this is by comparing the amount of information transmitted specifically about each cue when it was the target with the amount transmitted when it was not. This is shown in Figure 7 for the case in which the other cue was at the weaker of its two possible values. The same comparison was also made for the case in which the other cue was set at the stronger of its two values, with the same result: More information was transmitted by the response specifically about a given cue when it was the target than when it was not.

To test the significance of the effect of the task and its interactions, a five-way frequency analysis was performed using the variables response (matched to the orientation cue or not), length cue ( 5 or 15 pixels), orientation cue $\left(5^{\circ}\right.$ or $\left.15^{\circ}\right)$, task (length or orientation cue as the target), and observer (W.P. or A.W.). The simplest model producing a satisfactory fit [deviance $(24)=26.84, p>$ $.25]$ to the observed frequencies included only the twoway associations between response and length cue, response and orientation cue, and response and task. No other two-way or higher order associations were needed to account for the results. The association between response and length cue was significant [deviance (24) = $37.05, p<.05]$. The association between response and orientation cue was highly significant [deviance (24) = $67.92, p<.001]$, as was that between response and task [deviance $(24)=77.65, p<.001]$. Task instructions therefore had a large and highly significant effect on whether response was matched to a particular cue.

These results show that the contribution of the individual texture boundary cues to response is affected by the task context. This suggests that task context modulates information transmission gain, and this possibility was directly tested in Experiment 5.

\section{EXPERIMENT 5 Effects of Task Context on the Gain of Information Transmission About a Target Orientation Boundary}

Experiment 5 studied the effect of task instructions on the information transmission gain of an orientation cue. One task was to report the orientation of the texture bound- 

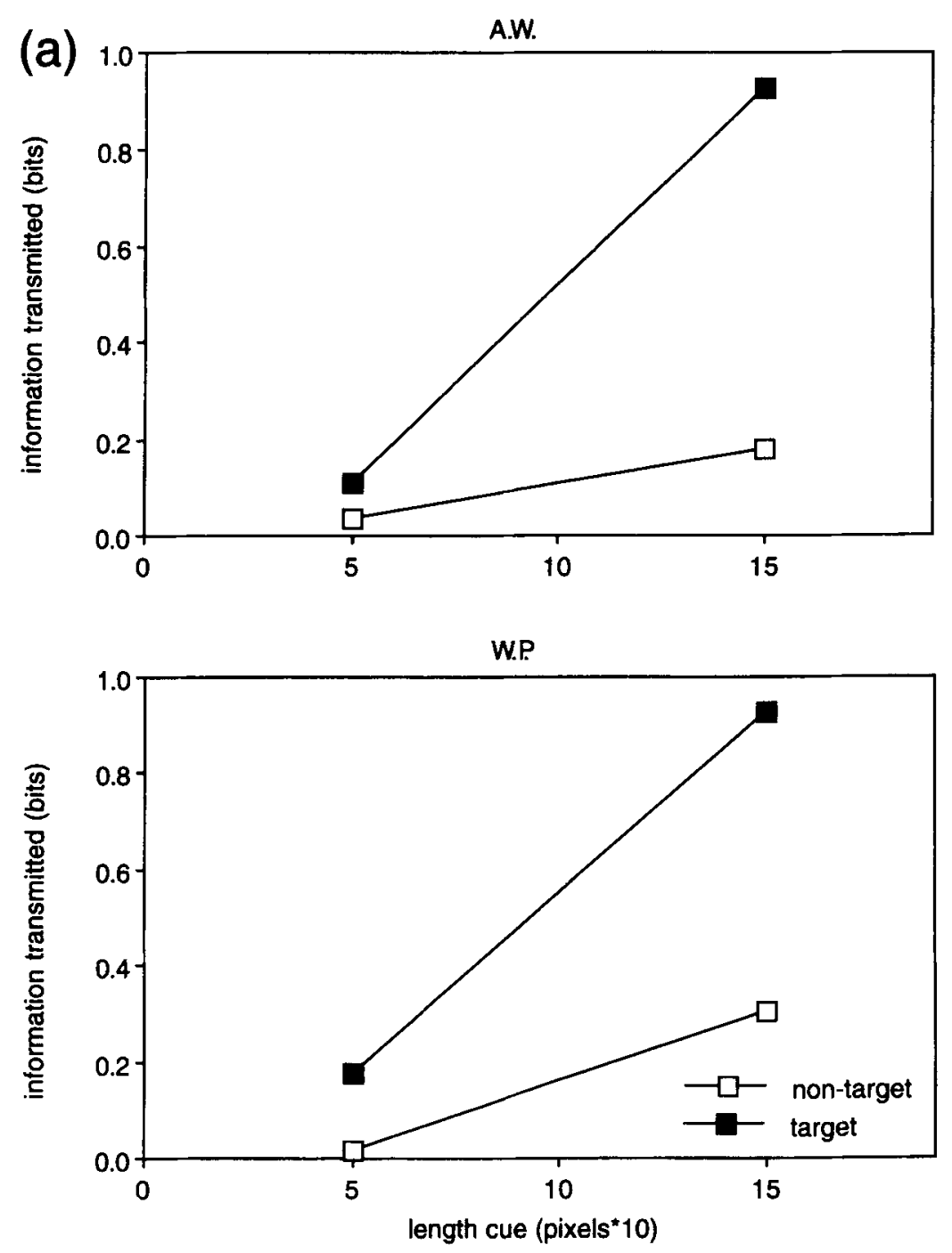

Figure 7a. Information transmitted specifically about the length boundary as a function of cue strength and of whether they were or were not specified as the target, for the 2 observers, A.W. and W.P.

ary formed by differences in the mean orientation of elements. The other task was to report the orientation of the texture boundary formed by differences in the mean length of elements. Four strengths of the orientation cue were chosen so as to study gain at low to medium strengths of this cue. A detectable but weak length cue was used so that it would not by itself dominate response and thereby obscure any modulatory effects of the task on processing of the orientation cue.

\section{Method}

W.P. and A.W. were again the observers. The texture boundary formed by the length cue was specified as the target in half of the blocks of trials, and the texture boundary formed by the orientation cue was specified as the target in the other half. One value of the length cue was used in all trials in which both cues were present (i.e., 0.5 pixels). Four values for the orientation cue were used: $2^{\circ}$, $5^{\circ}, 9^{\circ}$, and $15^{\circ}$. Trials were also run in which only one cue was present, it being the target. In these, the length cue was 0.5 or 1.5 pixels at random across one set of trials, and the orientation cue was $5^{\circ}$ or $15^{\circ}$ across another set. This gives four conditions in which the two cues were present and the length cue was the target, another four conditions differing only in that the orientation cue was the target, and four conditions with only the target cue being present. A full block of trials consisted of two main parts in which both cues were presented and in each of which one was specified as the target. The order in which the cues were specified to be the target was alternated across blocks. Within these blocks, each part was preceded and followed by a set of trials in which the cue specified as the target for that part of the block was presented alone. The probability that the two cues were coincident when both were present was .5. The observers performed 20 trials in each condition in each of five full blocks of trials, requiring about $30 \mathrm{~min}$ per block and providing 100 trials for each of the 12 conditions for each observer. 

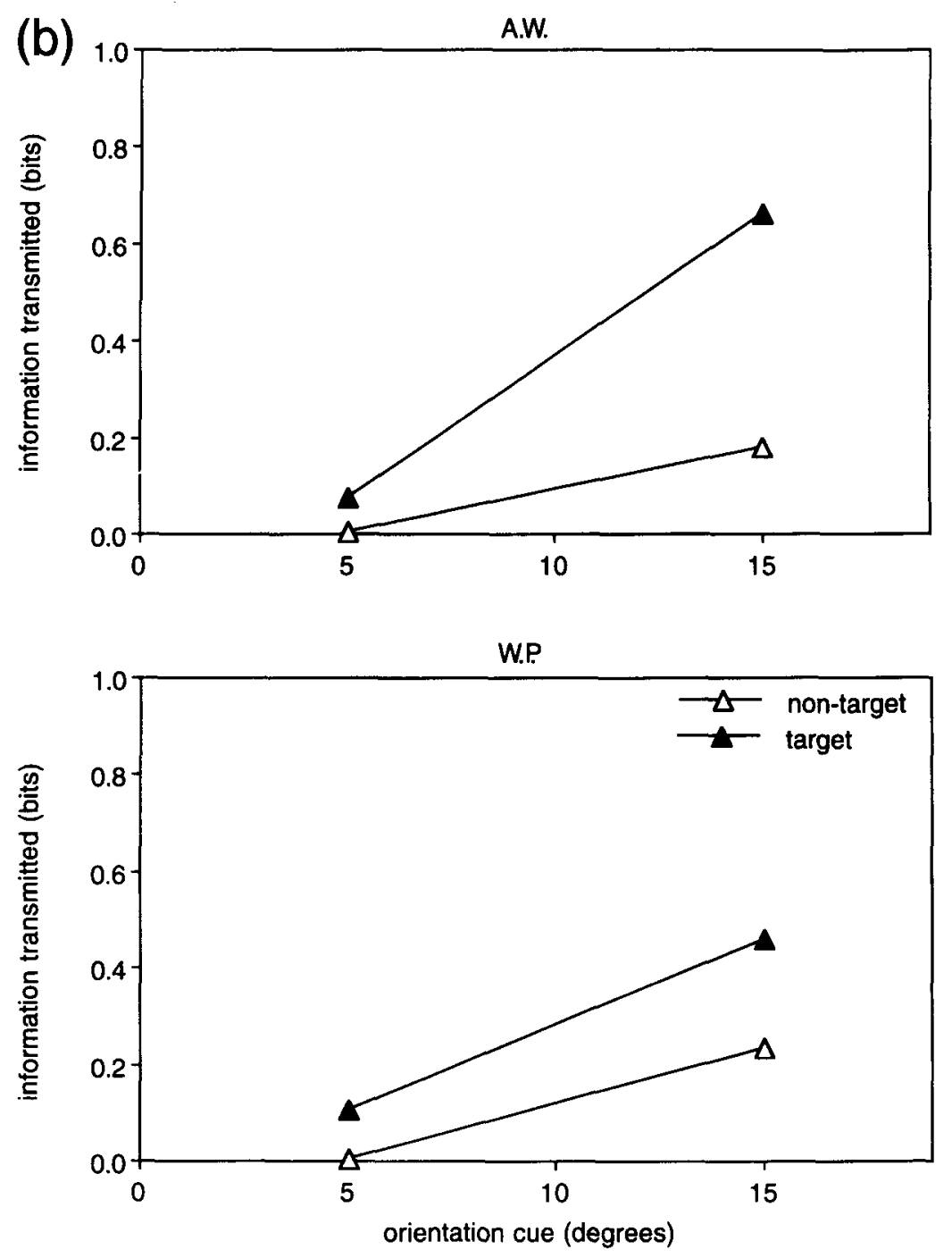

Figure $7 \mathrm{~b}$. Information transmitted specifically about the orientation boundary, as a function of cue strength and of whether they were or were not specified as the target, for the 2 observers, A.W. and W.P.

\section{Results and Discussion}

The percentages of responses that matched the target cue were again higher with a coincident nontarget cue than with an orthogonal nontarget cue. Task instructions had no effect on the percentages of responses that matched the orientation cue when it was at its two weakest values $\left(2^{\circ}\right.$ and $\left.5^{\circ}\right)$ but had a large effect when it was at its two stronger values $\left(9^{\circ}\right.$ and $\left.15^{\circ}\right)$. Estimates of $d^{\prime}$ for the orientation cue show that the rate at which it increased with the strength of that cue was much greater when it was the target than when it was not. For Observer W.P., $d^{\prime}$ rose from near zero with an orientation cue of $2^{\circ}$ to 4.65 with a cue of $15^{\circ}$ when it was the target but rose only to 1.95 when it was not. For Observer A.W., $d^{\prime}$ rose from near zero with a cue of $2^{\circ}$ to 4.50 with a cue of $15^{\circ}$ when it was the target but rose only to 1.06 when it was not. The effect of task instructions on the gain of response to the orientation cue is also clearly seen in the measures of information transmitted specifically about the orientation cue, as shown in Figure 8.

To test the significance of the effects of task instructions, a four-way frequency analysis was performed using the variables response (matched to the orientation cue or not), orientation cue $\left(2^{\circ}, 5^{\circ}, 9^{\circ}\right.$, or $\left.15^{\circ}\right)$, task (length or orientation cue as the target), and observer (W.P. or A.W.). The four-way interaction between these variables was required to produce a satisfactory fit to the observed frequencies, so no further simplification of the model was attempted. This four-way association was highly significant [deviance $(18)=169.6, p<.001$ ]. This implies that, though weak, the nontarget cue still had a significant effect. The form of the interaction can be clearly seen in Figure 8. The gain of information transmission about the orientation boundary was greater when it was specified 

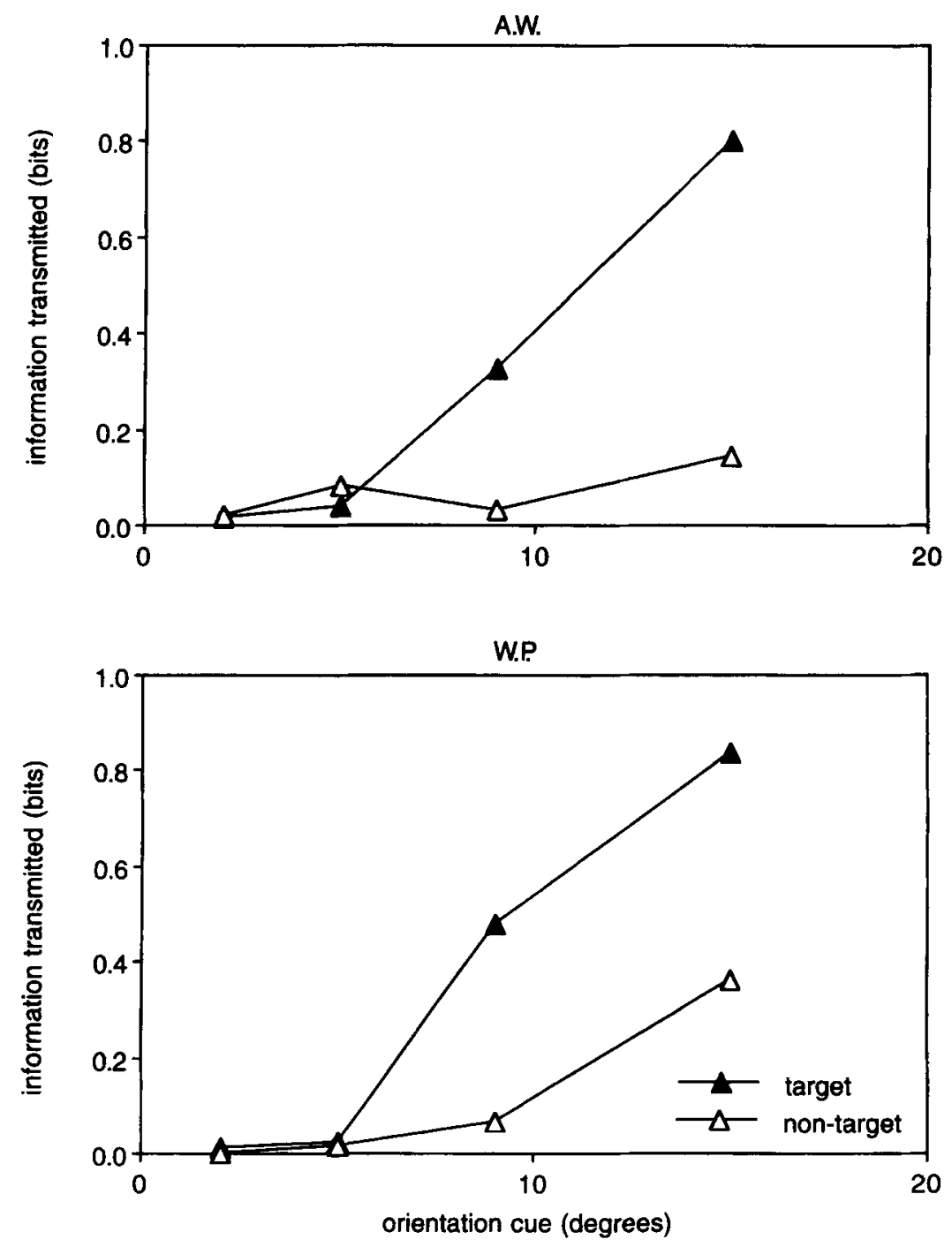

Figure 8. Information transmitted specifically about the orientation boundary, as a function of cue strength and of whether it was or was not specified as the target, for the 2 observers, A.W. and W.P.

as the target than when it was not, and this effect was greater for Observer A.W. than for Observer W.P.

These results provide evidence that task information can modulate the transmission of information about specific texture boundary cues, because they show that specifying a cue to be the target greatly increases the gain in the transmission of information specifically about that cue.

\section{GENERAL DISCUSSION}

The main outcomes are clear. A generalized texture boundary map signaling feature gradients summed across coincident texture cues and not distinguishing between them contributed to performance in these experiments.
Evidence for modulatory interactions between the separate texture cues used here was sought, but none was found. There was, however, clear evidence that task context can modulate the effects of the separate cues.

The results thus indicate that length and orientation texture boundary cues arising from the same region of the image are processed independently prior to their combination in a generalized map. We have sought evidence for the distinctive signs of a modulatory interaction between these texture cues in many other experiments not reported here. None have provided any reliable evidence for such an interaction. The majority of previous studies providing evidence for a generalized map used visual search or pop-out tasks. The results reported above sup- 
port the view that such a generalized map is relevant to situations in which the task is to detect a weak texture boundary that is displayed at an attended location for $1 \mathrm{sec}$. This suggests that its role extends beyond that of drawing attention to regions of space with steep texture gradients.

Our evidence for additive interactions between texture boundary cues is consistent with evidence for the use of multiple cues in texture edge localization paradigms (Landy, 1993; Rivest \& Cavanagh, 1996). Landy (1993) suggests that the weighting given to individual cues when combining them depends on their ecological reliability. The effects of task context in our experiments suggest that the weightings given to individual cues in determining response may, in addition, be subject to dynamic modifications that depend on strategic control processes.

Several reasons were outlined in the introduction for supposing that there are modulatory interactions between at least some stimulus cues, so it is worth asking why no such interaction was observed between the cues used here. One possibility is that only strong nontarget stimuli produce modulation. If so, a paradigm allowing the use of strong nontarget stimuli would be needed to provide evidence for modulation. Another possibility is that modulation may affect only the extent to which cues at-

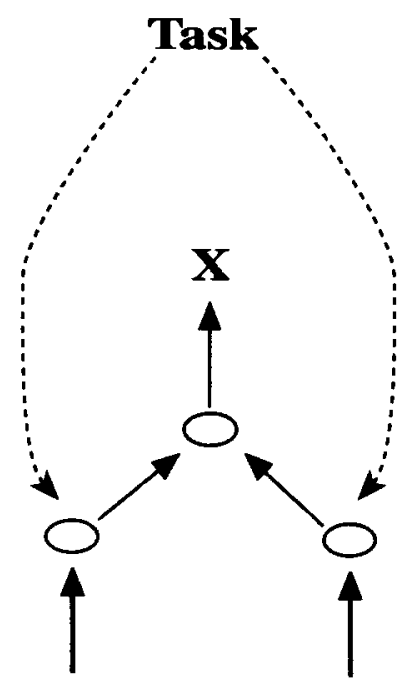

\section{Target Nontarget cue cue}

Figure 9. Interactions indicated by the results. Distinct texture boundary cues are summed to form a combined output in which the identity of the distinct cues that contribute to it are not distinguished. The gain of transmitted information for each cue is not directly modulated by the other cue, but it is modulated by the task context (as shown by dotted arrows). Transmission of information about the combined output, $X$, can also depend on the task, but that is not shown since our experiments were not designed to study such effects. tract attention. If so, visual search or pop-out tasks should provide evidence for modulatory interactions between these cues, even though the paradigm used here does not. Yet another possibility is that the particular cues used are insufficiently correlated in natural images and that their correlation within the experiment was insufficient to establish modulatory relations between them. If so, a more extended training period within the experiment may produce modulatory interactions, particularly if it includes some form of feedback reinforcing detection of the correlation. Finally, it may be that no modulatory interactions can be established between the streams processing these particular cues because there are no appropriate anatomical connections between them. Such connections may not be needed because, as argued by Phillips and Singer (1997), their main functions may be to allow interactions between processing streams that remain distinct and to dynamically group those signals that need to be treated as a whole at the next stage of processing. Neither of these may be required in the case of coincident texture cues, because they are not kept distinct at higher stages of processing and may be automatically grouped by anatomical feedforward convergence to those higher stages.

The conception of cortical computation argued for by Phillips and Singer (1997), and which is supported by a wide variety of empirical data, suggests that modulatory interactions between stimulus variables are common. The paradigm and information theory measures presented in this paper can be used to test for such modulatory interactions between other stimulus variables. If few can be found, then either modulation is less common than Phillips and Singer suggest or the criteria for modulation used here are inappropriate.

The failure to find evidence for modulatory interactions between stimulus cues in these experiments highlights the need to clarify terminology so as to distinguish the disparate senses in which something may be described as a "context." This term is often used to refer to any variable other than that specified by the experimenter to be the target. In this sense, the above experiments clearly demonstrate effects of "stimulus context," because they show large effects of stimulus variables other than the target. However, this does not imply that the target and nontarget variables influenced performance in fundamentally different ways, and, in this case, they did not. Modulatory effects, as defined here, affect performance in a way that differs fundamentally from that of the variables whose effects they modulate. Such effects can therefore be called "contextual effects" in the sense that they are a special kind of effect. The tests for this kind of effect do not depend on what is called the target.

One simple interpretation of the modulatory effects of task context is that information about the task modulates the contribution of the different cues to the generalized texture boundary map (Figure 9). This is in keeping with the findings of several studies of the effects of attention 
on activity in visual cortex (Desimone \& Duncan, 1995), which have shown that the contributions of different components of the receptive field inputs to pyramidal cells in various regions of the visual cortex are modulated by the current task. However, the results reported above do not necessarily imply that it is the input to the common map that is modulated by attention. It is possible that information about different cues can contribute to performance separately as well as through a generalized map and that their separate contributions are modulated by attention. The finding that attention can be directed toward one texture cue rather than another does imply, however, that it is not only to texture cues made salient via the generalized map (Nothdurft, 1992) that attention can be directed. This is because different texture cues are not distinguished within the generalized map.

A primary motivation for the present experiments was that the theory of Phillips and Singer (1997) implies that modulatory interactions are a fundamental component of cortical computation. The results reported here provide no evidence for modulatory interactions between length and orientation cues to texture boundaries when they arise from the same region of the image, but they do show that task contexts can modulate the effects of the different cues in these conditions. This supports suggestions (König, Chiang, \& von Stein, 1997; Tononi, Sporns, \& Edelman, 1997; Wright, 1997) that the theory of Phillips and Singer would be strengthened by an increased emphasis on internal sources of contextual modulation.

\section{REFERENCES}

Atkinson, J., \& Braddick, O. (1989). "Where" and "what" in visual search. Perception, 18, 181-189.

Attneave, F. (1954). Informational aspects of visual perception. Psychological Review, 61, 183-93.

Barlow, H. B. (1961). Possible principles underlying the transformations of sensory messages. In W. A. Rosenblith (Ed.), Sensory communication (pp. 217-234). Cambridge, MA: MIT Press.

Barrow, H., \& TEnEnbaum, J. (1978). Recovering intrinsic scene characteristics from images. In E. Riseman (Ed.), Computer vision systems (pp. 3-26). New York: Academic Press.

BECK, J. (1967). Perceptual grouping produced by line figures. Perception \& Psychophysics, 2, 491-495.

BECK, J. (1982). Textural segmentation. In J. Beck (Ed.), Organization and representation in perception (pp. 285-317). Hillside, NJ: Erlbaum.

Caelli, T., \& Moraglia, G. (1985). On the detection of Gabor signals and discrimination of Gabor textures. Vision Research, 25, 671-684.

Callaghan, T. C. (1984). Dimensional interaction of hue and brightness in preattentive field segregation. Perception \& Psychophysics, 36, 25-34.

Callaghan, T. C. (1989). Interference and dominance in texture segregation: Hue, geometric form, and line orientation. Perception \& Psychophysics, 46, 299-311.

Callaghan, T. C., Lasaga, M. I., \& Garner, W. R. (1986). Visual texture segregation based on orientation and hue. Perception \& Psychophysics, 39, 32-38.

Desimone, R., \& DunCan, J. (1995). Neural mechanisms of selective visual attention. Annual Review of Neuroscience, 18, 193-222.

FARELL, B. (1984). Attention in the processing of complex visual displays: Detecting features and their combinations. Journal of Experimental Psychology: Human Perception \& Performance, 10, 4064.
Friston, K. J., Buechel, C., Fink, G. R., Morris, J., Rolls, E., \& Dolan, R. J. (1997). Psychophysiological and modulatory interactions in neuroimaging. Neurolmage, 6, 218-229.

Friston, K. J., UngerLeider, L. G., Jezzard, P., \& Turner, R. (1995). Characterizing modulatory interactions between areas $\mathrm{V} 1$ and $\mathrm{V} 2$ in human cortex: A new treatment of functional MRI data. Human Brain Mapping, 2, 211-224.

GARNER, W. R. (1962). Uncertainty and structure as psychological concepts. New York: Wiley.

GARNER, W. R. (1974). The processing of information and structure. Potomac, MD: Erlbaum.

GiLberT, C. D. (1992). Horizontal integration and cortical dynamics. Neuron, 9, 1-13.

GrEen, M. (1991). Visual search, visual streams, and visual architectures. Perception \& Psychophysics, 50, 388-403.

Kay, J., Floreano, D., \& Phillips, W. A. (1998). Contextually guided unsupervised learning using local multivariate binary processors. Neural Networks, 11, 117-140.

König, P., Chiang, C., \& von Stein, A. (1997). Internal context and top-down processing. Behavioral \& Brain Sciences, 20, 691-692.

LANDY, M. S. (1993). Combining multiple cues for texture edge localization. In B. E. Rogowitz \& J. P. Allebach (Eds.), Human vision, visual processing and digital display $I V$ (Proceedings of the SPIE, 1993; pp. 506-513). Bellingham, WA: SPIE Press.

LANDY, M. S., \& BeRgen, J. (1991). Texture segregation and orientation gradient. Vision Research, 31, 679-691.

NothDURFT, H.-C. (1992). Feature analysis and the role of similarity in preattentive vision. Perception \& Psychophysics, 52, 355-375.

NothDURFT, H.-C. (1993). Saliency effects across dimensions in visual search. Vision Research, 33, 839-844.

Pashler, H. (1988). Cross-dimensional interaction and texture segregation. Perception \& Psychophysics, 43, 307-318.

PhILliPS, W. A., KAY, J., \& SMYTH, D. (1995). The discovery of structure by multi-stream networks of local processors with contextual guidance. Network, 6, 225-246.

Phillips, W. A., \& Singer, W. (1997). In search of common foundations for cortical computation. Behavioral \& Brain Sciences, 20, 657-722.

Poggio, T., Gamble, E., \& Little, J. (1988). Parallel integration of visual modules. Science, 242, 436-439.

Polat, U., Mizobe, K., Pettet, M. W., \& Norcia, A. M. (1998). Collinear stimuli regulate visual responses depending on cell's contrast threshold. Nature, 391, 580-584.

POLAT, U., \& SAGI, D. (1993). Lateral interactions between spatial channels: Suppression and facilitation revealed by lateral masking experiments. Vision Research, 33, 993-999.

Polat, U., \& SAGI, D. (1994). Spatial interactions in human vision: From near to far via experience-dependent cascades of connections. Proceedings of the National Academy of Sciences, 91, 1206-1209.

Rivest, J., \& CavanaGH, P. (1996). Localizing contours defined by more than one attribute. Vision Research, 36, 53-66.

SAGI, D. (1995). The psychophysics of texture segmentation. In T. V. Papathomas (Ed.), Early vision and beyond (pp. 69-78). Cambridge, MA: MIT Press.

SAGI, D., \& JULESZ, B. (1985). "Where" and "what" in vision. Science, 228, 1217-1219.

Scialfa, C. T., \& JofFe, K. M. (1995), Preferential processing of target features in texture segmentation. Perception \& Psychophysics, 57, 1201-1208.

Smyth, D., Phillips, W. A., \& Kay, J. (1996). Measures for investigating the contextual modulation of information transmission. Network, 7, 307-316.

Tabachnick, B. G., \& Fidell, L. S. (1989). Using multivariate statistics. New York: Harper Collins.

THEEuWEs, J. (1991). Cross-dimensional perceptual selectivity. Perception \& Psychophysics, 50, 184-193.

TheEUWES, J. (1992). Perceptual selectivity for color and form. Perception \& Psychophysics, 51, 599-606.

Tononi, G., Sporns, O., \& Edelman, G. M. (1992). Reentry and the problem of integrating multiple cortical areas: Simulation of dynamic integration in the visual system. Cerebral Cortex, 2, 310-335. 
Tononi, G., Sporns, O., \& Edelman, G. M. (1997). Information: In the stimulus or in the context? Behavioral \& Brain Sciences, 20, 698-701.

Troscianko, T., Davidoff, J., Humphreys, G., Landis, T., Fahle, M., Greenlee, M., Brugger, P., \& Phillips, W. A. (1996). Human colour discrimination based on a non-parvocellular pathway. Current Biology, 6, 200-210.

Victor, J. D., Conte, M. M., Purpura, K., \& Katz, E. (1995). Isodipole textures: A window on cortical mechanisms of form processing. In T. V. Papathomas, C. Chubb, A. Gorea, \& E. Kowler (Eds.), Early vision and beyond (pp. 99-107). Cambridge, MA: MIT Press.

WolfE, J. M. (1994). Guided Search 2.0: A revised model of visual search. Psychonomic Bulletin \& Review, 1, 202-238.
Wolfe, J., Cave, K., \& Franzel, S. (1989). A modified featureintegration model for visual search. Journal of Experimental Psychology: Human Perception \& Performance, 15, 419-433.

WRIGHT J. J. (1997). Local attractor dynamics will introduce further information to synchronous neuronal fields. Behavioral \& Brain Sciences, 20, 701-702.

Zipser, K., LAMme, V. A. F., \& SChiller, P. H. (1996). Contextual modulation in primary visual cortex. Journal of Neuroscience, 16, 73767389 .

(Manuscript received June 30, 1998; revision accepted for publication July 7,1999 .) 